\title{
L'IMPATTO DELLA PANDEMIA DI COVID 19 SULLA DIAGNOSI DI CANCRO IN UN SERVIZIO DI PATOLOGIA NEL SUD DEL BRASILE
}

\section{ARTICOLO ORIGINALE}

KLOCK, Julia Cristhina Monteiro ${ }^{1}$, Borges, Giuliano Santos², OGATA, Daniel Cury ${ }^{3}$, KLOCK, Clóvis ${ }^{4}$

KLOCK, Julia Cristhina Monteiro. Et al. L'impatto della pandemia di covid 19 sulla diagnosi di cancro in un servizio di patologia nel sud del Brasile. Revista Científica Multidisciplinar Núcleo do Conhecimento. Anno. 06, Ed. 11, Vol. 14, pp. 182-190. Novembre 2021. ISSN: 2448-0959, Link di accesso: https://www.nucleodoconhecimento.com.br/salute/servizio-di-patologia, $\quad$ DOI: 10.32749/nucleodoconhecimento.com.br/salute/servizio-di-patologia

\section{RIEPILOGO}

La pandemia di Covid-19 ha avuto un impatto significativo sulla vita delle persone. Uno di questi impatti può essere verificato nella diagnosi di altre malattie, in particolare il cancro. Problema: qual è stato l'impatto sulla diagnosi di nuovi casi di cancro nella regione coperta da un servizio di patologia nel sud del Brasile durante la pandemia? Obiettivo generale: valutare se c'è stata una diminuzione del numero di diagnosi di cancro in un servizio di patologia nel sud del Brasile durante la pandemia. Metodologia: Abbiamo analizzato i case report effettuati nei Laboratori del Gruppo Infolaudo, durante i primi mesi della pandemia (aprile e maggio 2020) e confrontati con gli stessi mesi del 2019, al fine di misurare l'impatto della pandemia sulla diagnosi di cancro in un servizio di patologia nel sud del Brasile. Risultati: Confrontando i periodi di aprile e maggio 2019 con lo stesso periodo del 2020, è stata notata una diminuzione del $34,2 \%$ delle procedure patologiche. Alcuni tipi di

\footnotetext{
${ }^{1}$ Studente di medicina - Università di Mogi das Cruzes. ORCID: 0000-0002-0834-193X.

2 Specializzazione in Oncologia, Laurea in Medicina. ORCID: 0000-0002-0737-7922.

${ }^{3}$ Dottore in Chirurgia, Master in Chirurgia, Specialità in Patologia, Laurea in Medicina. ORCID 0000-0001-88192155.

${ }^{4}$ Dottorando in Oncologia, Specialità in Patologia, Laurea in Medicina. ORCID 0000-0001-8456-0061.
}

RC: 103131

Disponibile in: https://www.nucleodoconhecimento.com.br/salute/servizio-di-patologia 
cancro, come il colon, l'esofago, il seno e la prostata, hanno avuto una significativa diminuzione delle diagnosi, essendo rispettivamente del 19,7\%, 45\%, 18,2\% e $37,7 \%$. Conclusione: con la chiusura di molte cliniche e cliniche nelle prime fasi della pandemia, c'è stata una diminuzione delle diagnosi di cancro nei laboratori esaminati.

Parole chiave: Pandemia, Covid 19, cancro, patologia.

\section{INTRODUZIONE}

L'Organizzazione Mondiale della Sanità (OMS) stima che entro il 2030 ci saranno 27 milioni di nuovi casi di cancro, 17 milioni di morti per la malattia e 75 milioni di persone che vivono con il cancro in tutto il mondo (INCA, 2020).

Il cancro è la principale causa di morte nei paesi sviluppati e in via di sviluppo (ALLGAR e NEAL, 2005). Tuttavia, alcuni tipi di cancro hanno un'alta probabilità di guarigione se vengono rilevati in una fase precoce e trattati in modo appropriato (AMBUSAIDI e AL-BALUSHI, 2012).

Ritardi nella diagnosi del cancro possono verificarsi durante la diagnosi, in base ai livelli di assistenza sanitaria: paziente, cure primarie e cure secondarie.Le diagnosi nelle malattie neoplastiche più avanzate possono verificarsi quando il paziente è lento a riconoscere e agire in relazione ai sintomi sospetti (ASCO, 2020). Questa bassa consapevolezza dei primi sintomi del cancro è considerata la ragione predominante per una presentazione tardiva, specialmente quando i sintomi sono di natura atipica (ASCO, 2020). Un'altra possibile barriera potrebbe essere correlata all'elevata domanda di servizi medici specializzati e potrebbe eventualmente ritardare la diagnosi, specialmente nei servizi pubblici (INCA, 2020).

In Brasile, secondo l'INCA, nel 2020 sono stati stimati 309.230 nuovi casi di cancro, negli uomini, il cancro alla prostata è stato responsabile del 29,2\% dei casi stimati. Nelle donne, dei 316.140 nuovi casi attesi, il tumore al seno corrispondeva al 29,7\%. Questi dati non includono il cancro della pelle non melanoma (GREEN et al., 2015). 
In un caso, il ritardo nella diagnosi del cancro può verificarsi su diversi livelli. II paziente può non riconoscere i sintomi sospetti di cancro o agire su di essi. II medico di base potrebbe non riconoscere i pazienti con sospetti sintomi di cancro e indagarli correttamente o indirizzarli in tempo.I pazienti con sospetto clinico, in cure secondarie, potrebbero non essere visti in tempo o potrebbero essere indirizzati alla specialità sbagliata.

Durante la pandemia di coronavirus, le misure preventive adottate, come: distanziamento sociale e quarantena, hanno influenzato la diagnosi iniziale dei malati di cancro. Pertanto, il sovraffollamento osservato in diversi ospedali ha reso impossibile diagnosticare e trattare correttamente questi pazienti.

In questo scenario, i dati del campione locale non sono in grado di verificare se la pandemia abbia davvero colpito questo gruppo di pazienti e le diagnosi di cancro.

In considerazione di ciò, il presente articolo ha come domanda guida: qual è stato l'impatto sulla diagnosi di nuovi casi di cancro nella regione coperta da un servizio di patologia nel sud del Brasile durante la pandemia? Con l'obiettivo generale di valutare se c'è stata una diminuzione del numero di diagnosi di cancro in un servizio di patologia nel sud del Brasile durante la pandemia.

Sono stati analizzati i dati dell'inizio della pandemia (aprile e maggio) del 2020, confrontandoli con i dati di aprile e maggio 2019, da un Servizio di Patologia della Regione Meridionale del Brasile (Gruppo Infolaudo) per riconoscere la situazione attuale e gli impatti derivanti dal periodo di lockdown sulla diagnosi di questo gruppo di individui.

Si tratta di uno studio epidemiologico trasversale e quantitativo del campione locale.

\section{MATERIALI E METODI}

Sono stati analizzati tutti i case report di aprile e maggio 2020 e aprile e maggio 2019, effettuati nei Laboratori del Gruppo Infolaudo, e confrontati tra loro. I periodi scelti sono stati definiti a causa dell'inizio delle misure di isolamento sociale e RC: 103131

Disponibile in: https://www.nucleodoconhecimento.com.br/salute/servizio-di-patologia 
lockdown. Tutti i pazienti inclusi in questo studio sono stati trattati nella regione foz del fiume Itajaí, Alto vale, Florianópolis e il nord e l'ovest del Rio Grande do Sul (Erechim e Cruz Alta).

Tutti i casi con diagnosi di cancro sono stati inclusi nello studio. Sono stati esclusi i casi che non avevano conferma della diagnosi di cancro. I dati raccolti sono stati incorporati nel programma Microsoft Office Excel® 2010 e i risultati sono stati presentati in numeri e percentuali assoluti. Si tratta di uno studio epidemiologico trasversale e quantitativo del campione locale.

\section{DISCUSSIONE}

Ritardi nelle cure primarie possono verificarsi nel riconoscimento, nell'indagine e nel rinvio di casi con sospetta neoplasia maligna (ASCO, 2020). Sebbene l'assistenza primaria sia il primo punto di contatto per i pazienti che utilizzano i servizi sanitari nazionali in diversi paesi del mondo, il ritardo nella diagnosi del cancro rimane un problema continuo a questo livello (JONES et al., 2007). Ci si aspetta che i medici di base identifichino i pazienti con possibile malattia in una fase precoce. Tuttavia, la diagnosi di cancro è relativamente rara per il medico di base, in quanto oltre l'80\% dei pazienti ha sintomi non specifici (HARFORD, 2011). In effetti, la preoccupazione principale di questi operatori sanitari è quella di differenziare la minoranza di pazienti che hanno bisogno di cure urgenti da quelli che probabilmente hanno condizioni autoli limitate (KAUFMAN et al., 2020).

Nel maggio 2020, l'American Society of Clinical Oncology (ASCO) ha pubblicato un rapporto speciale che raccomanda il rinvio di qualsiasi visita clinica e di qualsiasi screening del cancro o diagnosi e procedure correlate alla stadiazione se questo rinvio non rappresenta un rischio di progressione della malattia o peggioramento della prognosi (LONDON et al., 2020).

Alcuni studi internazionali dimostrano che la diminuzione della diagnosi di cancro nei primi mesi della pandemia è stata del $65,2 \%$ dei nuovi casi di cancro (LONDON et al., 2020). 
Lo screening di alcuni tipi di cancro è stato compromesso, con dati che mostrano che il cancro al seno e al colon e al retto sono stati i più colpiti rispettivamente con l'89,2 e l'84,5\% (MACLEOD et al., 2009).

In uno studio condotto nel Regno Unito, il lockdown ha causato la sospensione degli screening oncologici, compromettendo la diagnosi precoce di innumerevoli pazienti. Solo di questo, i pazienti con quadro clinico critico e sintomatico sono stati indirizzati all'intervento diagnostico. I record di cancro sono stati utilizzati nel Servizio Sanitario Nazionale (NHS) attraverso un database di ospedali con pazienti di età compresa tra 15 e 84 anni, con diagnosi di cancro al seno (35583), colon-retto (24975) e cancro esofageo (6744) nel 2010 con follow-up fino al 2014. Nei pazienti con neoplasia polmonare primaria (29305), il 2012 è stato utilizzato come anno di diagnosi e il 2015 come data finale di follow-up. Attraverso un diagramma di flusso per definire $i$ percorsi dei malati oncologici all'interno del NHS, è stata fatta una stima per valutare le conseguenze del ritardo diagnostico in questo gruppo di pazienti, su un periodo di 12 mesi, a partire da marzo 2020 (data di lockdown),contestualizzando con il suo impatto 1,3 e 5 anni dopo la diagnosi iniziale. In questa metodologia, sono stati considerati tre percorsi o flussi di questi pazienti, corrispondenti dallo scenario migliore a quello peggiore. Sulla base di ciò, è stato stimato l'impatto effettivo della sopravvivenza a 1, 3 e 5 anni dopo la diagnosi, calcolando così il numero totale di decessi attribuiti dal cancro e il numero totale di anni di vita persi, confrontando con i dati pre-pandemia.

In tutti gli scenari, un aumento dal $7,9 \%$ al $9,6 \%$ dei decessi per cancro al seno è stato stimato entro 5 anni dalla diagnosi, il che significa rispettivamente da 281 a 344 decessi in più. Nel cancro del colon-retto, l'aumento è stato dal $15,3 \%$ (1445) al $16,6 \%$ (1563) e nei tumori polmonari, questo aumento è stato del $4,8 \%$ (1235) al $5,3 \%$ (1372). Infine, l'aumento osservato nei pazienti con cancro esofageo è stato del $5,8 \%$ (330) al 6\% (342). Questi dati mostrano che c'è stato un aumento significativo dei decessi prevenibili nel Regno Unito, probabilmente a causa di misure restrittive e di isolamento sociale (MARINGE et al., 2020). 
Un altro studio pubblicato da Kaufman et al. (2020) ha osservato una riduzione del $40 \%$ dell'incidenza settimanale del cancro nei Paesi Bassi e del $75 \%$ nel Regno Unito dall'inizio della pandemia di covid-19. Questo studio ha utilizzato una metodologia simile alla nostra, valutando i record di un database da gennaio ad aprile 2019, rispetto allo stesso periodo del 2020. Hanno notato cali significativi nella diagnosi di neoplasie maligne, in situ e comportamento indeterminato. Durante la pandemia, il numero settimanale di casi è diminuito di oltre il $46 \%$ nei sei tipi più comuni di cancro, con variazioni del $24,7 \%$ nei casi di neoplasia pancreatica e del 51,8\% nei casi di seno (OMS, 2016).

Un altro articolo, pubblicato da ricercatori del SidneyKimmelCenter, di Philadelphia e del NHS, ha mostrato una riduzione dell' $89,2 \%$ nello screening del cancro al seno e una diminuzione dell' $85,5 \%$ delle colonscopie, che è uno strumento importante per la diagnosi precoce del cancro del colon-retto (VOSE, 2020).

Tutti i casi del Gruppo Infolaudo nei periodi di marzo e aprile 2020 sono stati confrontati con il periodo di marzo e aprile 2019. Sono stati selezionati i mesi di marzo e aprile, in quanto sono stati i primi mesi di lockdown.

Nel 2019, nei mese di aprile e maggio, i Laboratori del Gruppo Infolaudo hanno ricevuto 5524 procedure patologiche, di cui 4634 lesioni benigne e 890 lesioni maligne, pari al $16,1 \%$. Nel 2020, nello stesso periodo, ci sono state 3635 procedure, con 2.875 lesioni benigne e 760 lesioni maligne, che rappresentano il 20,9\% maligno. C'è stata una diminuzione del $34,2 \%$ delle procedure patologiche. (Grafico 1) 
Grafico 1: Diminuzione delle procedure patologiche in aprile e maggio 2020 rispetto allo stesso periodo del 2019.

\section{Lesões benignas X Lesões malignas}

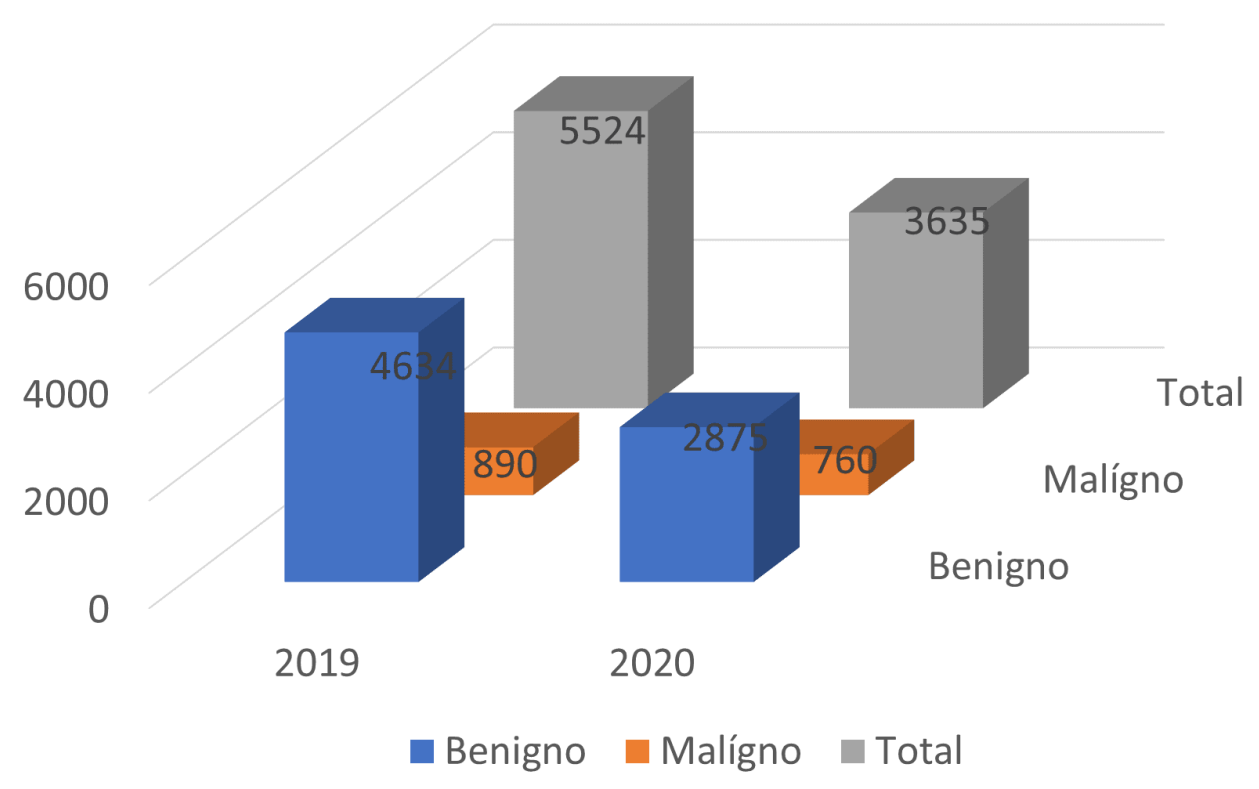

Fonte: autori

In generale, in tutte le diverse procedure, c'è stata una diminuzione della diagnosi, che si tratti di biopsie o campioni chirurgici. Alcuni tipi di procedure hanno avuto una diminuzione più marcata, come i principali interventi chirurgici elettivi che hanno richiesto il ricovero in ospedale del paziente.

Alcuni tipi di cancro (Grafico 2) hanno avuto una diminuzione più significativa, come il colon (19,7\%), l'esofago (45\%), la mammella $(18,2 \%)$ e la prostata $(37,7 \%)$.

Grafico 2: diminuzione delle diagnosi di cancro (per organo) più colpite in aprile e maggio 2020 rispetto allo stesso periodo del 2019. 


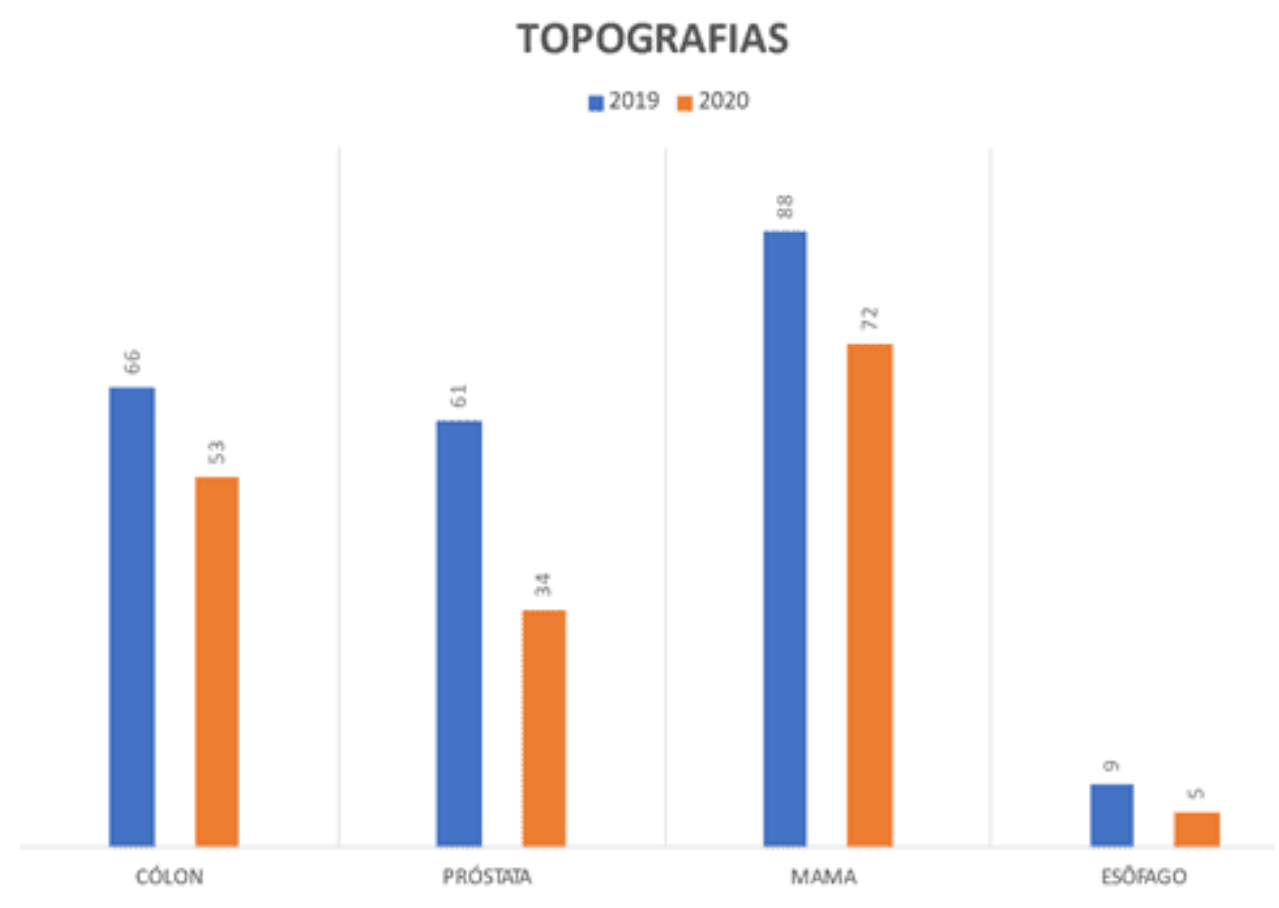

Fonte: autori.

La diminuzione principale era nei casi che avrebbero richiesto una procedura invasiva e nei casi di sedazione del paziente. Altri tipi, come i tumori della pelle, non sono diminuiti nello stesso periodo.

I blocchi chirurgici della maggior parte degli ospedali hanno anche limitato gli interventi chirurgici elettivi, a causa della necessità di lasciare i letti vuoti, in particolare i letti di terapia intensiva.

Con l'impatto della chiusura di molte cliniche e cliniche nelle prime fasi della pandemia, c'è stato un grande impatto su queste diagnosi. Oltre alla paura che i pazienti lasciano le loro case, che è stato anche uno dei fattori che hanno contribuito a questa diminuzione.

Questo studio ha alcune limitazioni. In primo luogo, è stato valutato solo l'impatto del blocco sulla diagnosi in un gruppo di laboratori, sebbene si tratti di un grande servizio. I risultati del presente studio, quindi, non sono necessariamente applicabili ad altri centri, quindi sarebbero necessari studi multicentrici per convalidare questi 
risultati su scala nazionale. È possibile che ciò abbia indotto un bias di selezione. Tuttavia, questo metodo di selezione dei pazienti era identico per tutti i periodi studiati, consentendo la comparabilità.

\section{CONSIDERAZIONI FINALI}

Tornando alla domanda giusta: qual è stato l'impatto sulla diagnosi di nuovi casi di cancro nella regione coperta da un servizio di patologia nel sud del Brasile durante la pandemia? Attraverso lo studio presentato, è stato possibile concludere che i dati analizzati nelle segnalazioni dei pazienti osservati nel Gruppo Infolaudo hanno mostrato che la diagnosi di alcuni tipi di cancro è diminuita nel periodo iniziale della pandemia nel 2020 con il periodo analizzato del 2019, dimostrando che la pandemia ha influenzato questo tipo di diagnosi. C'è stata anche una grande diminuzione del numero di biopsie e campioni chirurgici ricevuti nel periodo. Questa spiegazione può essere multifattoriale e può essere influenzata dalla chiusura dei servizi diagnostici come cliniche e settori ospedalieri, nonché dalla paura dei pazienti che cercano cure mediche.

\section{RIFERIMENTI}

ALLGAR, V. L.; NEAL, R. D. Delays in the diagnosis of six cancers: analysis of data from the National Survey of NHS Patients: Cancer. Br J Cancer, 2005.

AMBUSAIDI, A.; AL-BALUSHI, S. Educação em Saúde no Sultanato de Omã. In: Taylor, N. et al. (ed.). Educação em Saúde em Contexto. Editores Sense. 2012.

ASCO. American Society of Clinical Oncology. COVID-19 Patient Care Information. 2020. Disponível em: https://www.asco.org/asco-coronavirus-information/careindividuals-cancer-during-covid-19. Acesso em: 26 de jun. de 2020.

GREEN, T. et al. Cancer detection in primary care: insights from general practitioners. Ir. J Cancer, 2015. 
HARFORD, J. B. Breast-cancer early detection in low-income and middleincome countries: do what you can versus one size fits all. Lancet Oncol, 2011.

INCA. Instituto Nacional de Câncer. Estimativa 2020: incidência de câncer no Brasil. Rio de Janeiro: INCA, 2020.

JONES, R. et al. Alarm symptoms in early diagnosis of cancer in primary care: cohort study using General Practice Research Database. BMJ, 2007.

KAUfMAN, H. W. et al. Changes in the Number of US Patients With Newly Identified Cancer Before and During the Coronavirus Disease 2019 (COVID-19) Pandemic. JAMA Netw Open, 2020.

LONDON, J. W. et al. Effects of the COVID-19 pandemic on cancer-related patient encounters. JCO Clinical Cancer Inform, 2020.

MACLEOD, $U$. et al. Risk factors for delayed presentation and referral of symptomatic cancer: evidence for common cancers. Br J Cancer, 2009.

MARINGE, C. et al. The impact of the COVID-19 pandemic on cancer deaths due to delays in diagnosis in England, UK: a national, population-based, modelling study. Lancet Oncol, 2020.

VOSE, J. M. Delay in Cancer Screening and Diagnosis During the COVID-19 Pandemic: What Is the Cost? Oncology (Williston Park), 2020.

WHO. World Health Organization. Facts about cancer. 2016. Disponível em: www.who.int

Pubblicato: novembre 2021.

Approvato: novembre 2021. 